\title{
Numerical simulation of particle motion in the rotary flow in binoculars
}

\author{
Z.W. MU \& X.N. Zhang \& Y.Y. Li \\ College of Water Conservancy and Civil Engineering, Xinjiang Agricultural University, \\ Urumqi, Xinjiang
}

KEYWORD: DPM model; Concentric binoculars; Difference rotational inertia force, centrifugal force, Moving mesh

ABSTRACT: Using DPM model to simulate the particle with different size motion in the rotary flow in binoculars continuous, the sliding mesh used the concentric binoculars spiral flow simulation, and take the numerical simulation of the different particle motion in the flow to explore the motion characteristics and the force law. The simulation results show that when the single particle moving in rotating flow in the concentric binoculars, the smaller the speed, the increase tended distance from the center of rotation, and the centrifugal motion occurred. With the increase of the density, the difference rotational inertia force and the centrifugal force showed increase trend. The size of the particle size directly affects the value of the difference rotational inertia force and the centrifugal force, and showing positive correlation trends.

\section{INTRODUCTION}

For concentric binoculars spiral flow stress analysis of single particles, Ma Mingxiang has used the concentric binocular device to studied experimentally of the single particle motion in the flow. That single particle in a continuous rotation flow system is moving, the centrifugal force is an order of magnitude than the difference rotational inertia force in the magnitude. Zhang Xuening has used the DPM model to simulate the rotating flow in the concentric binoculars. She use the sliding mesh instead of the rotate inner wall to take the numerical simulation the single particle motion in the flow, and discussed the force situation of a fixed single particle in both speed. In this paper, for the particles' combinations of different sizes, different densities, different speed, it take a numerical simulation with the single particles motion in the system of the concentric binocular rotation.

\section{SIMULATION OVERVIEW}

The numerical simulation using the ANSYS FLUENT to simulate the single particle motion in the continuous rotary binoculars flow to establish the numerical model. Using the DPM model to track the single particle trajectory, and by capturing particle motion in different time periods to calculate the velocity of the particles, and compared the cylinder speed of the particle in each position.

2.1 Basic mathematical equations

Solid single particle motion in a rotary drum water flow presents complex, whose motional equation in two-dimensional is in line with the Newton's second law. Due to the limitations of two-dimensional conditions, we only consider resistance , additional mass force, centrifugal force and different rotational inertial force .

(1) Due to the change of the direction or speed the fluid resistance that single particle with the fluid in the flow process $F_{Z}$ is: 
$F_{Z}=\frac{\pi r_{P}^{2}}{8} C_{D} \rho u_{d}^{w}$

Where: $C_{D}$ is the drag coefficient, $u_{d}^{w}$ is the continuous phase fluid velocity component from a single particle, $r_{P}$ is the radius of a single particle.

(2) Single particle suffered mass forces $F_{J}$, when the particles as acceleration in the fluid, the fluid has inertia, the particle generation reaction. Its expression is:

$F_{J}=\frac{1}{2} \rho V_{d}\left(\frac{d u^{w}}{d t}-\frac{d u_{d}^{w}}{d t}\right)$

In the formulas: $u^{w}$ is the flow instantaneous velocity component, $V_{d}$ is the volume of a single particle.

(3) Single particle in the rotating water system suffered centrifugal force $F_{l}$ is:

$F_{l}=m \frac{v_{l}^{2}}{r_{l}}$

Where: $\mathrm{m}$ is the quality of solid single particles, $v_{l}$ is the radial velocity of solid single particles, $r_{l}$ is the distance from particle centroid to reference point.

(4) Single particle in the rotating system suffered difference rotational inertia force $F_{Y}$ is:

$F_{Y}=-2 m \vec{\omega} \times v_{d}$

Where: $\omega$ is the rotational angular velocity, $v_{d}$ is solid single particle with respect to the radial direction of the relative velocity of the continuous phase fluid.

2.2Basic mathematical equations

For the simulation of the single particle motion in the rotary flow at the concentric binoculars, we need to use the sliding mesh instead of the rotary inner wall, and the circle between the binoculars used for interface when establish the model use the GAMBIT. Numerical simulation in accordance with the test device which given solid single particle motion in the spiral flow at the concentric binoculars, using the GAMBIT 13.0 to build the two-dimensional model, and meshing and quadrilateral meshes. Figure 1 is a computing grid chart. Grid settings using a sliding mesh, the yellow curve between the binoculars ie data exchange surface. Reuse ANSYS FLUENT in the CFD to setting the DPM model. For the single particle motion in the rotation system with the different density and size in the binoculars rotating flow was simulated. Table 1 shows the simulated motion conditions.

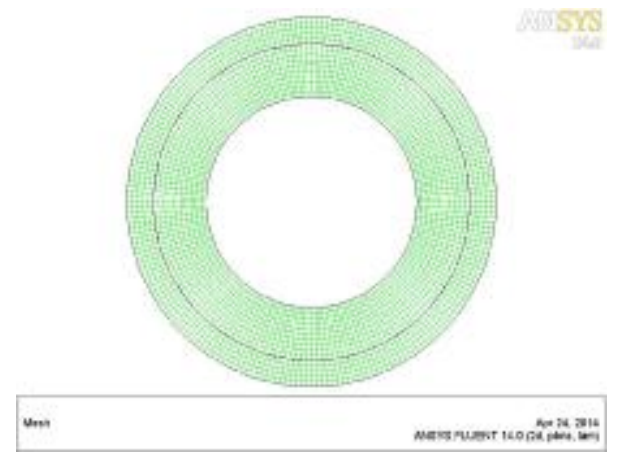

Figure1 Computing grid 
Table 1 Simulation category Settings

\begin{tabular}{|c|c|c|c|c|}
\hline $\begin{array}{l}\text { Working } \\
\text { conditions }\end{array}$ & $\begin{array}{l}\text { Density } \\
\left(\mathrm{kg} / \mathrm{m}^{3}\right)\end{array}$ & $\begin{array}{c}\text { Particle } \\
\text { (m) }\end{array}$ & $\begin{array}{l}\text { Speed } \\
(\mathrm{r} / \mathrm{min})\end{array}$ & $\begin{array}{c}\text { Quality } \\
\text { (g) }\end{array}$ \\
\hline 1 & 937 & 0.002 & 16 & 0.004 \\
\hline 2 & 1500 & 0.004 & 16 & 0.050 \\
\hline 3 & 1500 & 0.004 & 30 & 0.050 \\
\hline 4 & 937 & 0.004 & 30 & 0.030 \\
\hline 5 & 2000 & 0.004 & 16 & 0.067 \\
\hline 6 & 2000 & 0.004 & 30 & 0.067 \\
\hline
\end{tabular}

\section{NUMERICAL SIMULATION ANALYSIS}

Numerical simulation of single particle motion with different particle size, density and speed in continuous rotation cylinder use the DPM model. Figure 2 shows flow in a rotating drum continues to rotate with the same density, the same particle size, Single particle distance from the center of rotation in different speed at the same time. The figure shows, with the same diameter, the same density and the different speed conditions, speed from $\omega=16 \mathrm{r} / \mathrm{min}$ in ascending order to $\omega=35 \mathrm{r} / \mathrm{min}$, the smaller the speed at the same time, the greater the distance from the center of rotation, the occurrence of eccentric exercise, it can be proved that when a single particle occur centrifugal movement, speed is smaller, the faster the centrifugal tendencies the more obvious, but relative to the monocular rotation of the flow law is more obvious.

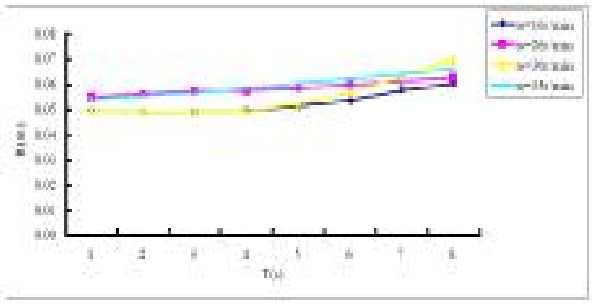

Figure2 Single particle distance from the center of rotation in different speed at the same time 
Figures 3 and 4 indicates that when a single particle density is $\rho=937 \mathrm{~kg} / \mathrm{m}^{3}$, a particle size is $d=0.004 \mathrm{~m}$, rotation speed respectively is $\omega=16 \mathrm{r} / \mathrm{min} 、 26 \mathrm{r} / \mathrm{min} 、 35 \mathrm{r} / \mathrm{min}$, the comparison of the single particle suffered centrifugal force and difference rotational inertial force. Figure 3 shows that when the speed is $\omega=16 \mathrm{r} / \mathrm{min}$, the centrifugal force of magnitude in a transition phase, that is the centrifugal force generated by $F=0.3701 \times 10^{-6} \mathrm{~N}$ increased to $F=1.83671 \times 10^{-6} \mathrm{~N}$, magnitude increased one level, other speeds are in the single particle suffered centrifugal force is $F=1.0 \times 10^{-6} \mathrm{~N}$ above. As the speed increases, the centrifugal force tended to increase, the same speed centrifugal force over time tended to increase. Figure 4 shows that when the $\omega=30 \mathrm{r} / \mathrm{min}$, single particle suffered different rotational inertia force in value from the $F=-0.7867 \times 10^{-6} \mathrm{~N}$ increased to $F=-2.1306 \times 10^{-6} \mathrm{~N}$, magnitude increased one level, other speed, the single particle suffered difference rotational inertia force is $F=0.1 \times 10^{-6} \mathrm{~N}$ above, and as the speed increases. It can be inferred that when $16 \mathrm{r} / \mathrm{min} \leq \omega \leq 30 \mathrm{r} / \mathrm{min}$, the centrifugal force and difference rotational inertia force in value on the same order of magnitude, and when $\omega \geq 30 \mathrm{r} / \mathrm{min}$, the centrifugal force is bigger an order of magnitude than the difference rotational inertia force.

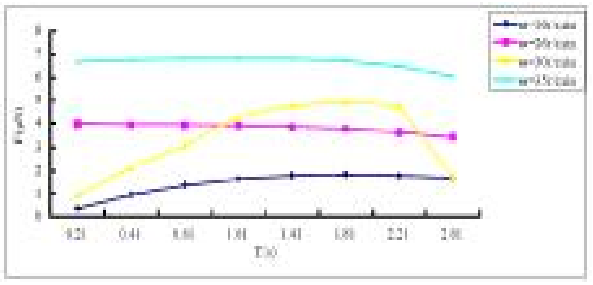

Figure3 Single particle suffered centrifugal force comparison of different speeds the flow in the cylinder rotating water in first working condition

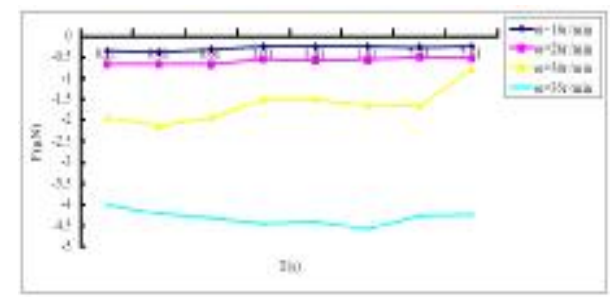

Figure4 Single particle suffered differences in rotational inertia force comparison of different speeds the flow in the cylinder rotating water in first working condition

Figures 5-7 shows that the single particle with the same speed and particle size, different densities of the force, then the single particle speed is $\omega=16 \mathrm{r} / \mathrm{min}$, particle size is $d=0.004 \mathrm{~m}$, density respectively is $\rho=937 \mathrm{~kg} / \mathrm{m}^{3} 、 1500 \mathrm{~kg} / \mathrm{m}^{3} 、 2000 \mathrm{~kg} / \mathrm{m}^{3}$. The figure shows that single particle with the same speed, the same particle size and the different density is moving in the rotary flow in the concentric binoculars, in the process of the single particle density increased from $\rho=937 \mathrm{~kg} / \mathrm{m}^{3}$ to $\rho=2000 \mathrm{~kg} / \mathrm{m}^{3}$, as the density increase, the fluid resistance, the difference rotational inertia force and the centrifugal showed a increasing tendency, the false quality force close to zero, the fluid resistance is much large than the other three force. The smaller the density, the smaller the gap between the four force of the single particle, the greater the density, the greater the gap between the force of the single particle. 


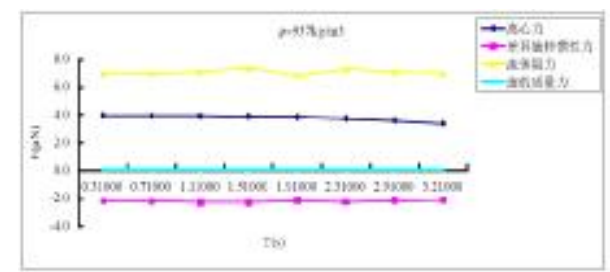

Figure 5 Different particle density of single grain motion in the drum rotating flow $\operatorname{stress}\left(\rho=937 \mathrm{~kg} / \mathrm{m}^{3}\right)$

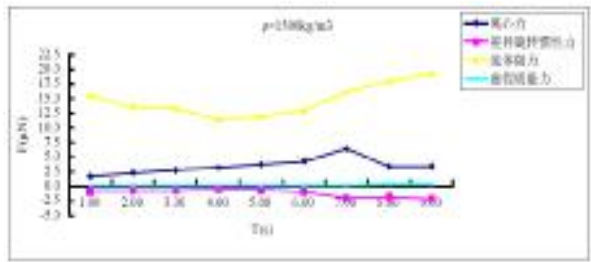

Figure 6 Different particle density of single grain motion in the drum rotating flow $\operatorname{stress}\left(\rho=1500 \mathrm{~kg} / \mathrm{m}^{3}\right)$

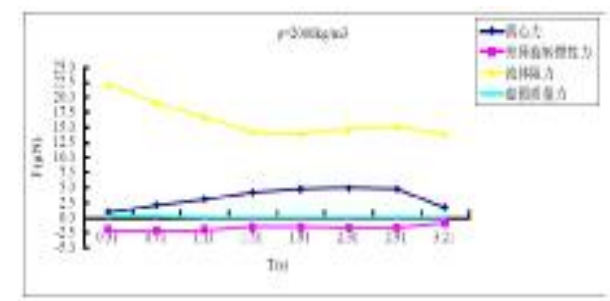

Figure 7 Different particle density of single grain motion in the drum rotating flow $\operatorname{stress}(\rho=2000 \mathrm{~kg} / \mathrm{m} 3)$

Figure 8 - 10 are the force contrast about the single particle movement with different size, same speed and same density. In this case single particle speed $\omega$ is $16 \mathrm{r} / \mathrm{min}$, particle size $d$ is $0.002 \mathrm{~m}, 0.004 \mathrm{~m}$, the density is $937 \mathrm{~kg} / \mathrm{m}^{3}$. Figure 8 shows that single particles with different particle sizes is moving in flow movement in a binoculars concentric rotation. Under the same speed and the same densities, the single particle's centrifugal trends which the $d$ is $0.004 \mathrm{~m}$ is faster than $d$ is $0.002 \mathrm{~m}$. Figure 9 shows that a single particle size of $\mathrm{d}$ is $0.004 \mathrm{~m}$ which suffered centrifugal force is bigger than $d$ is $0.002 \mathrm{~m}$ at the same time. Figure 10 shows that a single particle size of $d$ is $0.004 \mathrm{~m}$ which suffered difference rotational inertia force is much larger than $d$ is $0.002 \mathrm{~m}$ at the same time, and it is explain that the size of the mass of particles affect the size of the difference rotational inertia force.

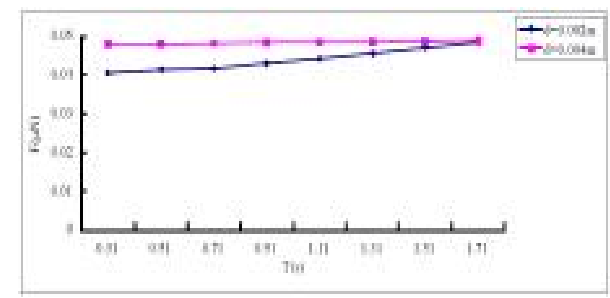

Figure 8 Different particle size of single grain in the drum rotating flow distance from the center of rotation $\left(\rho=937 \mathrm{~kg} / \mathrm{m}^{3}\right)$ 


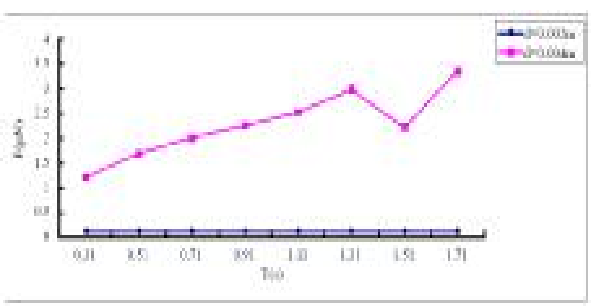

Figure 9 Different size of single particle motion in the drum rotating flow stressed centrifugal force $\left(\rho=937 \mathrm{~kg} / \mathrm{m}^{3}\right)$

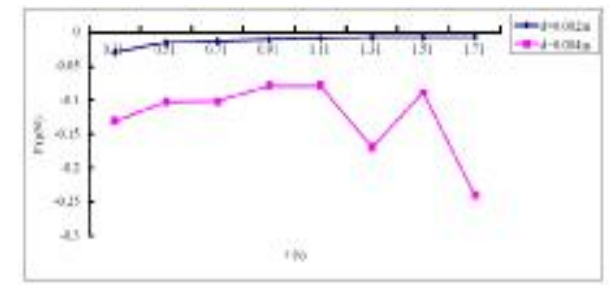

Figure 10 Different size of single particle motion in the drum rotating flow stressed differences in rotational inertia force $\left(\rho=937 \mathrm{~kg} / \mathrm{m}^{3}\right)$

\section{CONCLUSION}

(1) When the single particle with same density, the different speed is moving in the rotating flow at the concentric binoculars, with the speed smaller, the larger trend of the distance from the center of the rotation, then occur eccentric exercise. The single particles with the same speed, the same particle size, and the different density, with the increase of the density, the fluid resistance, differential rotation inertial force and the centrifugal force showed an increasing trend in the number, the false quality power is close to zero. The size of the particle size directly affects the value of the difference rotational inertia force and the centrifugal force, and showing positive correlation trends.

(2) when the single particle is moving in a continuous rotation concentric binoculars with rotation flow, the greater the speed, the greater the centrifugal force, the greater the difference rotational inertia force. When the speed is $16 \mathrm{r} / \mathrm{min} \leq \omega \leq 30 \mathrm{r} / \mathrm{min}$, the centrifugal force and difference rotational inertia force in value in the same order of magnitude, the single particles suffered centrifugal forces is $\mathrm{F} \geq 1.0 \times 10-6 \mathrm{~N}$ in the value. When the speed is $\omega \geq 30 \mathrm{r} / \mathrm{min}$, the centrifugal force is an order of magnitude than the difference rotational inertia.

\section{acknowledgements}

This work is financially supported by the National Natural Science Foundation of China (11162018). The authors would like to thank the colleagues' help in hydraulic laboratory.

\section{References}

[1] Xuening Zhang. Numerical simulation of the inertia force function in difference rotational motion[D]. Xinjiang Agricultural University, 2014.

[2] Mingxiang Ma. Experimental study of the inertia force function in difference rotational motion[D]. Xinjiang Agricultural University, 2013. 
[3] Zuobing Chen, Shou Li, Lijun Li. Simulation of two-phase flow within the preheater field by the DPM model[J]. Mechanical Engineering \& Automation, 2007, 2(141):51-55.

[4]Kuok DC,Ball KS. Taylor2Couette flow with buoyancy:Onset of spiral flow[J].Phys. Fluids,1997,9 (10):2872-2884.

[5] Man Zhang, Sheng Li. Study on propeller performance calculating based on sliding mesh[J]. Ship \& Ocean Engineering, 2013, 42(5):25-29.

[6] Zhiyan Zhang. Experimental study and numerical simulation of rotational coriolis force in small-scale solid-liquid two-phase flow [D]. Xinjiang Agricultural University, 2011. 